\title{
FROM THE THIRD GENEVA CONFERENCE ON PERSON-CENTERED MEDICINE
}

\section{The Third Geneva Conference on Person-centered Medicine: Collaboration across Specialties, Disciplines and Programs}

\author{
Juan E. Mezzich MD MA MSc PhD ${ }^{a}$ and Andrew Miles MSc MPhil PhD ${ }^{b}$ \\ a Deputy Editor-in-Chief, International Journal of Person Centered Medicine, Professor of Psychiatry, Mount Sinai School of Medicine, \\ New York University, USA, President, International Network for Person-centered Medicine \\ b Editor-in-Chief, International Journal of Person Centered Medicine, Professor of Clinical Epidemiology and Social Medicine, Medical \\ School, University of Buckingham, UK
}

\section{Keywords}

Communication, cultural and social diversity, ethics, Geneva conferences, human right to health, low and middle income countries, measurement, people-centered healthcare, personalized diagnosis, person-centered medicine, person-centered surgery, primary care, social determinants of health suffering

\section{Correspondence address}

Prof. Juan E. Mezzich, Professor of Psychiatry, Mount Sinai School of Medicine, New York University, Fifth Avenue and $100^{\text {th }}$ Street, Box 1093, New York NY 10029, USA. E-mail: juanmezzich@aol.com

Accepted for publication: 3 February 2011

\section{Introduction}

The Geneva Conference on Person-centered Medicine that took place in May 2010 represented an affirmation of an annual process initiated in May 2008. It also achieved the participation and support of a record number of international institutions, 22 of them, most notably the World Health Organization, which became a formal coorganizer of the event. The Third Geneva Conference followed the inaugural Geneva Conference of May 2008 [1] and the Second Geneva Conference of May 2009 [2] as landmark events in a process of building an initiative on Medicine for the Person through the collaboration of major global medical and health organizations and a growing group of committed international experts [3, 4].
The Third Geneva Conference took place on May 3-5, 2010 at the Marcel Jenny Auditorium of the Geneva University Hospital and in the Executive Board Room of the World Health Organization. It was organized by the International Network for Person-centered Medicine (INPCM), the World Medical Association (WMA), the World Organization of Family Doctors (Wonca), and the World Health Organization (WHO), in collaboration with the International Alliance of Patients' Organizations (IAPO), the International Council of Nurses (ICN), the International Federation of Social Workers (IFSW), the International Pharmaceutical Federation (FIP), the Council for International Organizations of Medical Sciences (CIOMS), the World Federation for Mental Health (WFMH), the World Federation of Neurology (WFN),

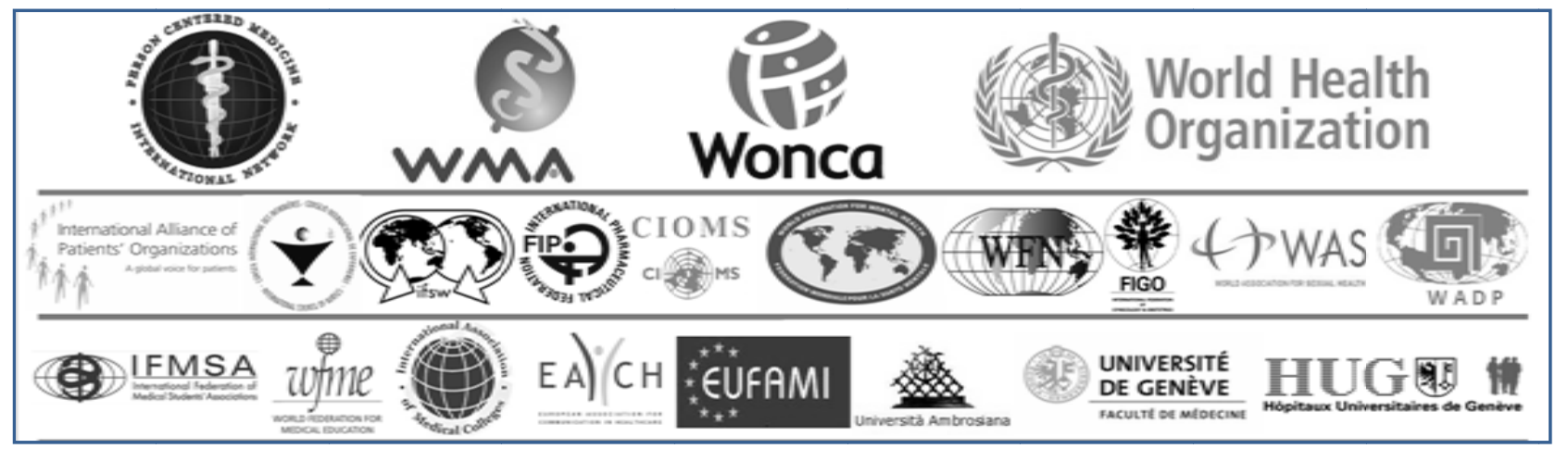

Fig 1: Logos of the institutions collaborating in the organization of the Third Geneva Conference on Person-centered Medicine 
the International Federation of Gynecology and Obstetrics (FIGO), the World Association for Sexual Health (WAS), the World Association for Dynamic Psychiatry (WADP), the International Federation of Medical Students' Associations (IFMSA), the World Federation for Medical Education (WFME), the International Association of Medical Colleges (IAOMC), the European Association for Communication in Health Care (EACH), the European Federation of Associations of Families of People with Mental Illness (EUFAMI), Ambrosiana University, Geneva University and the Paul Tournier Association. The logos of these institutions are presented in Figure 1. The Third Geneva Conference on Person-centered Medicine, under the overall theme of Collaboration across Disciplines, Specialties and Programs examined, through a set of sessions, the guiding value of person- and peoplecenteredness, ethical aspirations, basic communication skills, fundamental clinical care activities, the challenge of surgical and intensive care procedures, the vicissitudes of the life cycle and the implications of cultural diversity. The Conference Core Organizing Committee was composed of J.E. Mezzich (INPCM President and World Psychiatric Association President 2005-2008), J. Snaedal (World Medical Association President 2007-2008), C. van Weel (World Organization of Family Doctors President 2007-2010), I. Heath (Royal College of General Practitioners President), M. Botbol (WPA French Member Societies Association President), I. Salloum (WPA Classification Section Chair) and W. Van Lerberghe (Director of the WHO Department for Health System Governance and Service Delivery). Also collaborating organizationally were $O$. Kloiber (WMA Secretary General), A.M. Delage (WMA Secretariat), R. Kawar (WHO), and J. Dyrhauge (WHO). Financial or in-kind support for the Conference was provided by 1) the International Network for Person-centered Medicine (core funding), 2) the World Health Organization (covering invited participants' travel and accommodation expenses, a conference reception, and some secretarial and logistic services), 3) University of Geneva Medical School (auditorium services and coffee breaks), 4) Paul Tournier Association (a conference reception and the conference dinner for a group of invited participants), 5) The World Medical Association (local secretariat and printing services and support to extend the conference dinner to all participants) and 6) Participants' registration fees.

The Conference was opened by authorities of the University of Geneva Medical School, the Director of the WHO Department for Health System Governance and Service Delivery representing the WHO Assistant Director-General for Health Systems and Services, the President of the World Medical Association (WMA) and the members of the Organizing Committee. The opening address was delivered by the INPCM President, who presented a progress report on the INPCM's first months of existence emerging from the Second Geneva Conference. He touched on the establishment of a governing Board and initial organizational bases, development of an active publications program including a journal supplement with the edited papers from the First Geneva Conference, a well visited website, an institutional logo and the organization of the Third Geneva Conference.

\section{Sessions and symposia of the Third Geneva Conference}

\section{The first session}

The first session of the scientific program involved a symposium on person-centered medicine and primary health care organized by WHO. The key speaker was the Director of the WHO Department for Health System Governance and Service Delivery who presented arguments on why measuring person-centered medicine and people-centered care is vital. His presentation was commented on by general practitioners, academics and patient representatives. Importantly, the need to develop procedures for appraising the extent to which person- and people-centered care take place emerged as a clear recommendation.

\section{The second session}

A symposium on ethics and the person-centered approach constituted the second session. It started with an examination of the problems derived from reifying disease and restrictively considering numerical data which undermine full attention to subjective experience and the suffering person. The second presentation argued that attention to the social determinants of health is crucial for advancing human rights and ethics in health care. The final paper presented an African perspective including references to local concepts (ubuntu and batho pele), suggesting the value of placing people first, respect for diversity, and that what is good for the person is more important than what is good for his health.

\section{The third session}

The third session was a symposium on basic communication skills, a topic of increasing interest for person-centered care. Discussed first was an overview of research on communication behaviors which critically influence health care process and outcome. Such behaviors include providing room for a patient's story, exploring emotional cues, showing empathy and framing information and advice in a positive way, as well as optimizing outcomes through patient enablement, control, reassurance and adherence to jointly decided care plans. The second presentation pointed out that adequate person-centred communication is a cornerstone of good clinical practice and requires dedicated training and that the content of 
person-centeredness can vary depending on context and culture. It included interactive discussions attending to literature-based guidelines and participants' views. The third presentation on clinical teaching reviewed interviewing educational technologies, while consistently focusing upon person-centered principles.

\section{The fourth session}

The fourth session examined central clinical care activities from a person-centered perspective. The first presentation on personalized diagnosis suggested the need for a paradigmatic shift by focusing on both ill and positive health and the whole person and reported on international surveys and focus groups yielding salient recommendations for improving diagnostic systems. The second reviewed treatment plans as the written record of shared decisional and interactive processes between patients and clinicians, aimed at achieving desired life goals beyond the illnesses that threaten hopes and dreams. A third presentation charged that most contemporary medical treatment is focused on relief of acute symptoms of illness, rather than on the promotion of health and wellbeing and that specific procedures are emerging to facilitate the latter. The final presentation commented on current clinical services with constrained incentives based on volume rather than persons' values and reviewed evolving person-centered medical home models that demonstrate the challenges and rewards of transforming practices and which are gaining acceptance from health professionals, business leaders and policy makers.

\section{The fifth session}

The fifth session involved a panel discussion on special initiatives for person-centered care presented by representatives of international organizations of patients ("focus on the whole person, not just the disease") and medical students ("holistically seeing the person as a whole and not a sum of parts"), Italian ("forming PCM clinical teachers") and British ("need for a medicine of the whole person") universities, the World Federation for Mental Health ("treating the whole person concerning both physical and mental health"), psychodynamic ("selfreflection and self-monitoring of transference and countertransference feelings in daily clinical work") and public health ("global strategy for introduction of the PCM model”) programs, and INPCM projects on personcentered diagnosis ("a new model with related regional and national developments") and informational platforms ("to facilitate INPCM internal and external communication and full range of activities”).

\section{The sixth session}

The sixth session, a symposium on the team approach in person-centered health care emblematic of the
Conference's overall theme, was presented by officers from the global organizations of family doctors, nurses, social workers and pharmacists. For the President of the World Organisation of Family Doctors, responsiveness to the person's needs and values, continuity of care and team work based on common values and objectives, are at the core of person-centered medicine. The International Council of Nurses representative proposed that health systems be redesigned to optimize nursing contributions to health teams in general and to person-centered care in particular. According to the representative of the International Federation of Social Workers, these professionals bring emphases on contextualization and the patient's empowerment to person-centered team work. Finally, the representative of the International Pharmaceutical Federation highlighted the specific expertise that pharmacists can bring to collaborative practices in a variety of hospital and ambulatory settings and to adherence to care programs.

\section{The seventh session}

Seventh in the core program was a symposium on personcentered care in the context of surgical and intensive procedures. First discussed was person-centered surgery, which reviewed the importance of dialogue under time pressures, the need for understanding the person's condition and avoiding harmful procedures. Next, a presentation from the International Federation of Gynecology and Obstetrics reviewed the enormous development of multiple marker screening in early pregnancy which has led to more individualized informed consent decision making and counselling, as well as to health care system efficiencies. Finally considered were experiences at a Mongolian hospital intensive care unit where simple procedures such as providing a protective gown and conducting auscultation with body positions that afford greater patient privacy, seemed to enhance personcentered and more effective care.

\section{The penultimate session}

Next was a symposium on life cycle and personcentered care. It started with a presentation on personcentered pediatric care, which emphasized the uniqueness of every child, the importance of attending to his physical, emotional, social and spiritual needs through primary, secondary and tertiary prevention. A discussion of old-age person-centered care followed, which pointed out that personal lifestyle and historical patterns of diseases influence the presentation of symptoms and needs. It also noted that clinical care should pay special attention to abilities and disabilities to decide on a care plan, which should be designed considering the patient's wishes and aspirations. Completing this symposium was an examination of human development as fundamental to defining a person and person-centered care. Such 
definition lies at cross-roads between changes and continuity, maturation and personal history.

\section{The closing session}

The last symposium of the core conference dealt with cultural and social diversity in person-centered care. The role of culture in the conceptualization and experience of illness and positive health, as well as for effective health communication, was considered first. A second presentation reviewed the socioeconomic implications of comprehensive diagnosis, treatment and research, particularly in lesser-developed countries. Health policies based on the assessment of positive health and personcentered care were noted as promising to deal with the less resourced and more vulnerable sectors of the population. The last paper referred to the abundant documentation on the profound impact of gender on clinician-patient interactions across many countries and medical conditions and in terms of diagnosis, treatment as well as patient adherence and patient satisfaction and noted that this information seems to have been largely ignored in general health care planning.

\section{WHO, INPCM and people-centered care in low and middle income countries}

After the core conference and as the last session of the whole event, a special meeting was held at the WHO Executive Board Room focused on people-centered care in low and middle income countries. After opening words from the WHO Assistant Director General for Health Systems and Services and the INPCM President, a set of presentations highlighted experiences in implementing people-centred services in several low- and middle-income countries: El Salvador, Malaysia, Rwanda, Thailand, and the United Republic of Tanzania. The proceedings of this important session were subsequently published by WHO [5] and have been comprehensively analyzed by Miles [6].

After the individual country presentations, the Director of the WHO Department for Health System Governance and Service Delivery formulated comments recognizing the importance of the reports for person- and peoplecentered care and pointing out the need for advances in systematic conceptualization and measurement. An ensuing roundtable discussion on future avenues for making health care more people-centered across the world was chaired by the Director of the WHO Department for Human Resources for Health, and had as panelists the Secretary General of the World Medical Association, a psychiatry professor from India, a primary care and public health professor from the United Kingdom, and a patient/user consultant. Comments were also offered by a number of conference participants including the President of the World Medical Association. After a vigorous and interactive general discussion, conclusions articulated by the Assistant Director General for Health Systems and Services highlighted the importance of the event for advancing people-centeredness and the recent World Health Assembly resolution on the renewal of primary health care [7].

\section{Conclusion of the Third Geneva Conference}

A conference closing session offered summary comments and a consideration of next steps. These included broadening the engagement of health organizations, academic institutions and experts across the world; further construction of the International Network for Personcentered Medicine, its institutional identity, governance and operational structure; upgrading of the INPCM Website, informational base and clearinghouse functions; continuing publications in major journals and development of an international journal of person-centered medicine; research projects on diagnosis, clinical care and public health; increasing collaboration with WHO, based on 2009 World Health Assembly resolutions promoting peoplecentered care; and planning for a Fourth Geneva Conference on Person-centered Medicine in early May 2011.

\section{References}

[1] Mezzich J E, Snaedal J, Van Weel C, Heath I. (2010). Person-centered Medicine: A Conceptual Exploration. International Journal of Integrated Care, Supplement.

[2] Mezzich J E. (2009). The Second Geneva Conference on Person-centered Medicine. World Medical Journal 55: 100-101.

[3] Mezzich J E, Snaedal J, van Weel C, Heath I. (2010). Toward Person-Centered Medicine: From Disease to Patient to Person. Mount Sinai Journal of Medicine 77: 304-306.

[4] Mezzich J E, Snaedal J, van Weel C, Heath I. (2009). The International Network for Person-centered Medicine: Background and First Steps. World Medical Journal 55: 104-107.

[5] WHO (2010). Technical report on People-centered Care in Low and Middle Income Countries. Department of Health System Governance and Service Delivery, Third Geneva Conference on Person-centred Medicine May 5, 2010.

[6] Miles A. (2011). Tailoring care to individuals and populations within resource-poor settings: A review and commentary on the World Health Organisation Report PeopleCentred Care in Low and Middle Income Countries. International Journal of Person Centered Medicine 1: 1, 113-118.

[7] World Health Organization: Resolution WHA62.12. Primary health care, including health system strengthening. In: SixtySecond World Health Assembly, Geneva, 18-22 May 2009. Resolutions and Decisions. Geneva, 2009 (WHA62/2009/REC/1), Page 16. 